\title{
A Framework for Enhanced QoS Support in IEEE802.11e Networks
}

\author{
G. Cecchetti \\ gabriele@sssup.it
}

\author{
A. L. Ruscelli \\ anna@gandalf.sssup.it
}

\author{
ReTiS Lab. \\ Scuola Superiore S. Anna \\ Pisa, Italy
}

\begin{abstract}
IEEE 802.11 based WLANs have became popular, but they can only provide best effort services and so they are poorly suitable for multimedia applications. Recently IEEE 802.11e standard has been proposed to support quality of service. The new standard introduces a so-called Hybrid Coordination Function containing two medium access mechanisms: contention-based channel access and controlled channel access.

In this paper we propose a novel framework to better support QoS guarantees for multimedia applications. It comprises QoS Manager, Admission Control, Enhanced Scheduler, Predictor and Feedback System. The scheduler adopted supports real-time applications, variable packet sizes and variable bit rate traffic streams. We show that this framework is suitable to be used by applications requesting Application Level Contracts which will be translated in Resource Level Contracts to the scheduler subsystem. The QoS manager component is able to dynamically manage available resources under different load conditions.
\end{abstract}

\section{Keywords}

WLAN, QoS Management, Scheduling Algorithm.

\section{INTRODUCTION}

The increasing spreading of multimedia wireless transmissions has produced a growing interest in Quality of Service (QoS) support for Wireless Local Area Networks (WLANs) technology. Examples of applications range from standard Internet services, such as Web access, to real-time services with strict latency/throughput requirements, such as multimedia video and voice over IP (VoIP). With the high demands and varying requirements of these applications, there is a need to support QoS in WLAN. The wireless medium presents space and time varying characteristics differently from what it happens for the wired ones. Wireless access

Permission to make digital or hard copies of all or part of this work for personal or classroom use is granted without fee provided that copies are not made or distributed for profit or commercial advantage and that copies bear this notice and the full citation on the first page. To copy otherwise, or republish, to post on servers or to redistribute to lists, requires prior specific permission and/or a fee.

MobiMedia'06, Second International Mobile Multimedia

Communications Conference, September 18-20, 2006, Alghero, Italy Copyright 2006 ACM 1-59593-516-9/06/09...\$5.00 networks are subject to fast changes in signal to interference plus noise ratio (SINR) due to phenomena like path loss, shadowing, multipath fading, signal attenuation and interference. SINR, in turn, affects the bit error rate (BER) experienced by the wireless endpoints. In this environment channel capacity varies over time and space, especially when the stations are on the move. It turns out that the variability of available radio resources does not allow the network to provide hard QoS guarantees. Instead, the network must provide soft QoS guarantees constrained by a minimum channel quality. Some of these guarantees regards: delay, delay jitter, packet loss ratio, throughput, bandwidth.

While most commercialized WLAN products nowadays are based on the IEEE 802.11 standard [1], which is designed for best effort services, its current medium access control (MAC) specification offers little QoS support. As a step toward providing QoS support in WLAN, the 802.11 Working Group has developed a new protocol, IEEE 802.11e [2], to provide differentiation mechanisms at the medium access control (MAC) layer. A number of studies has evaluated the new standard by both analytical evaluation [14], as well as simulation [10] [12] [5], and has demonstrated the usefulness of the proposed mechanisms of 802.11e. Although 802.11e enhances the current access mechanisms, several scheduling algorithms were proposed to improve the QoS provisioning [7] [11] [13] [8].

In this article we first describe the various $802.11 \mathrm{~b}$ MAClayer mechanisms to access the medium and their limits, then we introduce the 802.11e QoS-enabled MAC mechanisms including its performance evaluations and most significative alternatives to its reference scheduler. Finally we present a novel framework to provide a comprehensive Hard and Soft QoS support for multimedia traffic streams with some discussion. We specifically focus on its components: QoS Manager, Admission Controller, Scheduler, Predictor and Feedback mechanism. The techniques presented in this paper improve QoS support adapting the parameters available in the $802.11 \mathrm{e}$ mechanisms. We show that such approach handles time-varying network conditions, heterogeneous traffic streams, VBR streams and it manages efficiently link layer resources.

\section{THE IEEE 802.11b STANDARD MAC}

The early standard IEEE 802.11 specifies two medium access control (MAC) mechanisms: the mandatory Distributed Coordination Function (DCF) and the optional Point Coor- 
dination Function (PCF) [1].

\subsection{Distributed Coordination Function (DCF)}

DCF works as a "listen-before-talk" scheme based on Carrier Sense Multiple Access with Collision Avoidance (CSMA/CA) where stations listen to the medium to determine when it is free and it provides distributed control. Collision is prevented by two mechanisms, one physical and one virtual. According the former, if a station that has packets to send senses the medium is busy, it will defer its transmission and initiate a backoff counter. The backoff counter is a uniformly distributed random number between 0 and Contention Window $(\mathrm{CW})$. Once the station detects that the medium has been free for a duration of DCF Inter-Frame Space (DIFS), it starts a backoff procedure (i.e., decrementing its backoff counter as long as the channel is idle). If the backoff counter has reduced to zero and the medium is still free, the station begins to transmit. According to the virtual mechanism, if the medium becomes busy in the middle of the decrement, the station freezes its backoff counter and resumes the countdown after deferring for a period of time, which is indicated by the so-called Network Allocation Vector (NAV), stored in the winning station's packet header. It is possible that two or more stations begin to transmit at the same time. In such a case, a collision occurs. Collisions are inferred by no acknowledgment (ACK) from the receiver. After a collision occurs, all the involved stations double their CWs (up to a maximum value, CWmax) and compete to gain control of the medium next time. If a station succeeds in channel access (inferred by the reception of an ACK), the station resets its $\mathrm{CW}$ to $\mathrm{CWmin}$.

DCF does not provide QoS supports but supplies best effort service as all stations operate with the same channel access parameters, they have the same medium access priority and there is no stream differentiation.

\subsection{Point Coordination Function (PCF)}

$\mathrm{PCF}$ provides contention-free transmission. In $\mathrm{PCF}$ time is divided into superframes. A superframe includes a Contention Period (CP), where DCF is used, and a ContentionFree Period (CFP), where PCF is used. A superframe starts with a beacon management frame transmitted by the Access Point (AP), which acts as a point coordinator. The time used by the AP to generate beacon frames is called Target Beacon Transmission Time (TBTT), which is announced in the previous beacon frame. PCF uses the PCF Inter-Frame Space (PIFS), which is longer than a Short Inter-Frame space (SIFS) but shorter than DIFS, to provide point coordinators higher priority in medium access than DCF stations. During the CFP, the AP polls its associated stations according to a predetermined order called polling list (usually in a round-robin manner). No station is allowed to transmit unless it is polled. If there is no pending transmission in a polled station, the response is a null frame containing no payload. The CFP ends when the AP sends a CF-end message. If the CFP terminates before all stations have been polled, the polling list will be resumed at the next CFP cycle from the previous stopping point. If the AP receives no response from a polled station after waiting for a PIFS, it will poll the next station or end the CFP. In this way, no idle period longer than a PIFS occurs during a CFP.

PCF does not make available adapted QoS guarantees. Mo-reover PCF has the unpredictable beacon delay prob- lem: a beacon has to be delayed if there is an unfinished DCF frame at the end of the previous superframe. Another problem is that it is very difficult to predict the transmission time of a polled station because the polled station can transmit a frame of any length between 0 and the size of the maximum MAC Service Data Unit (MSDU).

\section{THE IEEE802.11e STANDARD MAC}

The new standard IEEE802.11e introduce a new coordination function called the Hybrid Coordination Function ( $\mathrm{HCF})$ which multiplexes between two medium access modes: a distributed scheme called Enhanced Distributed Channel Access (EDCA) and a centralized scheme called HCF Controlled Channel Access (HCCA).

\subsection{Enhanced Distributed Channel Access (EDCA)}

EDCA is a channel access mode which provides prioritized QoS and enhances the original DCF by classifying traffic through the introduction of Access Categories (ACs). Each AC has its own transmission queue and its own set of channel access parameters. The most important ones are: Arbitrary Inter-Frame space (AIFS) which is minimum time interval for the medium to remain idle before starting backoff; Contention Window (CWmin and CWmax) which sets backoff interval; Transmission Opportunity (TXOP) limits is the maximum duration for which a node can transmit after obtaining access to the channel. Using these parameters, when data arrives from higher layers it is classified and placed in the appropriate AC queue. Then an internal contention algorithm is used to calculate the total backoff time for each AC. The AC with the smallest backoff wins the internal contention and uses this backoff value to contend externally for the wireless medium. Nodes with higher priority can access the channel earlier than other nodes and prioritized flows have the advantage of longer channel access with their TXOP.

\subsection{HCF Controlled Channel Access (HCCA)}

HCCA provides a centralized polling scheme to allocate gua-ranteed channel access to traffic flows based on their QoS requirements. It uses a QoS-aware Hybrid Coordinator (HC) which is usually located at the QoS Access Point (QAP) in infrastructured WLANs and it provides polled access to the wireless medium. In order to be included in the polling list of the HC, a QoS Station (QSTA) must send a QoS reservation request using the special QoS management frame which contains flow information, such as mean data rate, mean packet size, MAC service data unit size and maximum tolerable delay. Each individual flow needs one particular reservation request and it is classified and assigned to one of eight Traffic Streams (TS) of that QSTA.

TS's parameters are collected by using a Traffic Specification (TSPEC). HC aggregates every TSPEC of QSTA's TSs and determines the values of parameters needed by the transmission itself: Service Interval (SI) and TXOP. SI is the time duration between successive polls for the node and it is a submultiple of the $802.11 \mathrm{e}$ beacon interval duration. TXOP is the duration of each node based on the mean application data rates of its requested flows.

After a PIFS, HC gains control of the channel and within Controlled Access Phase (CAP) it polls the QSTAs according to its polling list. This list is generated by a scheduler. 
The 802.11 e does not specifies this scheduler but just offers some guidelines to design it. Moreover it provides a reference scheduler that is compatible with the use of link adaptation and it respects the minimum performance requirements.

The maximum time spent in HCCA for each SI is limited by the dot11CAPMax variable and the total controlled access time in a beacon interval is limited by dot11CAPRate. The duration of the controlled access period can be limited using these parameters and the effect of controlled access mode on traffic flows in contention access mode can be bounded.

\section{802.11e SCHEDULING ALGORITHMS}

Several studies has been done to verify performances of reference scheduler [10] [12] [5]. According to them, for every QSTA, fixed SI and TXOP based on mean values of the transmission parameters are useful for Constant Bit Rate TS, while they does not reflect the fluctuation of Variable Bit Rate TS. Particularly reference scheduler performances are evaluated using heterogeneous traffic stream like VoIP (G729 codec), video stream (MPEG4 codec) and burst "best effort" data stream. Some alternative algorithms introduce the following features: a) variable SI and/or TXOP, b) feedback based mechanism, c) queue length model.

\subsection{Scheduling Estimated Transmission Time - Earliest Due Date (SETT-EDD)}

This algorithm [9] limits the amount of time during which the stations control the wireless medium, it improves the performance of the scheduler and it enhances its flexibility. It uses the mean TXOP as a guideline for allocating time and uses a token bucket scheme of time units or TXOP timer to allow nodes to vary their TXOP over time according to their needs. The TXOP timer of station $\mathrm{j}$ increases at a constant rate equal to $\mathrm{TDj} / \mathrm{mSIj}$ (where $\mathrm{mSIj}$ is minimum SI of jth QSTA), which corresponds to the total fraction of time the station can spend in polled TXOPs. The TXOP timer has a maximum value equal to MTDj (where MTDj is the Maximum Time Duration of jth QSTA). The time spent by a station in a polled TXOP is deducted from the TXOP timer at the end of the TXOP. The station can be polled only when the value of the TXOP timer is greater than or equal to $\mathrm{mTDj}$, which ensures the transmission of at least one packet at the minimum PHY rate. The authors also propose to change the service interval for each node based on the traffic profile and use Earliest Deadline First (EDF) to determine the polling order. If the due time to poll a station is $t$, the next poll shall be issued on a time t' that satisfies the relation: $t+m S I<t^{\prime}<t+M S I$. Time instant $\mathrm{t}+\mathrm{mSI}$ is the instant after which the next poll can be done, equivalent to the release time in the real-time scheduling theory. Time instant $t+$ MSI is the maximum time by which the next poll has to be done, or deadline time.

It has been shown that the proposed flexibility in the scheduler for voice and video traffic leads to significant reduction in average transmission delay (up to 33 percent) and packet loss ratio (up to 50 percent).

\subsection{Fair HCF (FHCF)}

FHCF [4] tries to improve the fairness both of CBR and VBR flows by assigning variable TXOPs. These are computed using queue length. Actually FHCF is composed of two schedulers: the QAP scheduler estimates the varying queue length for each QSTA before the next SI and compares this value with the ideal queue length. The QAP scheduler uses a window of previous estimation errors for each TS in each QSTA to adapt the computation of the TXOP allocated to that QSTA. Because sending rate and packet size can change, this estimation can not be accurate. After this comparison QAP computes the additional requested time (positive or negative) for each TS of each QSTA and reallocates the corresponding TXOP duration. Then, the node scheduler located in each QSTA can redistribute the unused time among its different TSs since the TXOP is always allocated to a whole QSTA. It computes the number of packets to transmit in the TS and time required to transmit a packet according to its QoS requirements. Later, according to its allocated TXOP, it evaluates the remaining time that can be re-allocated. This is possible since each QSTA knows its TS queue size at the beginning of polling phase and it is able to estimate its queue length at the end of TXOP and the requested additional time for TS.

Performance study indicates that FHCF provides good fairness while supporting bandwidth and delay requirements for a large range of network loads and, because it uses to allocate TXOP the mean sending rate of VBR applications instead of the maximum sending rate usable for the standard $\mathrm{HCF}$ scheme, it may recover much time and more flows can accepted in HCCA.

\subsection{Feedback Based Dynamic Scheduler (FBDS)}

FBDS [3] assigns dynamically the TXOP according to queue length estimation while SI remains fixed. All the QSTAs which compose the communication system and its transmission queues are regarded as a system whose balance is perturbed by new incoming flows. The FBDS scheduler, which uses HCF, behaves as a closed loop controller which restores this balance by bandwidth recovering. This is possible due queue length information sent by each QSTA through a 8-bit subfield of QoS Control Field. Moreover the closed loop system uses a discrete time model which permits to estimate queue length at beginning of new CAP phase and so it acts as compensation system against errors produced by channel perturbations not previewed by the scheduling algorithm.

This algorithm guarantees the delay bounds required by audio/video applications in presence of very broad set of traffic conditions and networks loads by using a control system action which ensures a maximum delay for queuing new frames.

\section{THE PROPOSED FRAMEWORK}

We present a contract based scheduling framework that is suitable to integrate QoS support provided by IEEE $802.11 \mathrm{e}$ standard for applications with tightening guarantees and temporal boundaries. This framework represents high level abstraction that lets practitioners concentrate on the specification of the application requirements. The contract model is the mechanism that we have chosen for the application to dynamically specify its own set of complex and flexible execution requirements. From the application perspective, the requirements of an application component are written as a set of a service contracts for different resources, which are negotiated with the underlying implementation. To accept a set of contracts the proposed system has to check, as part of the negotiation, if it has enough resources to guarantee 
all the specified minimum requirements while keeping guarantees on all the previously accepted contracts negotiated by other application components. If a result of this negotiation is accepted, the system will reserve enough capacity to guarantee the minimum requested resources and it will adapt any spare capacity available to share it among the different contracts that have specified their desire or ability for using additional capacity. The contract also contains Quality of Service tuning parameters that may be used by QoS manager.

Its architecture is composed by QoS manager, admission control, predictor, enhanced scheduler and feedback system (See Fig.1).

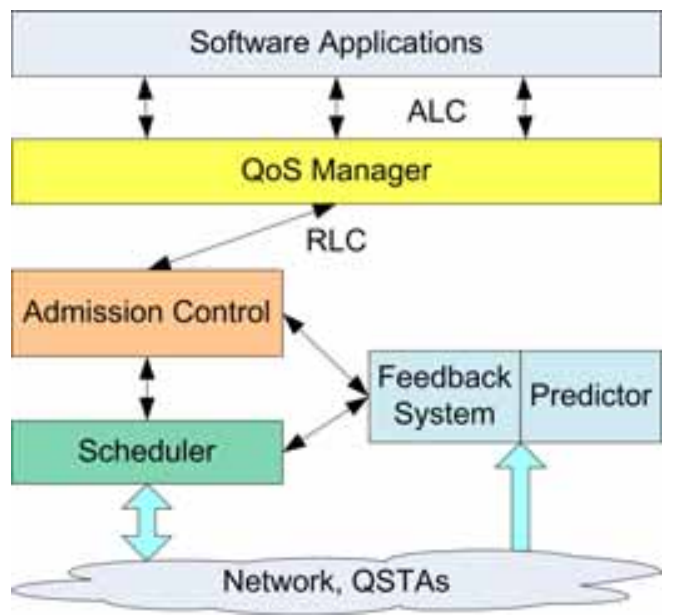

Figure 1: The Proposed Framework

QoS Manager [6] is a middleware layer that mediates between application and underlying components of this framework. Different applications specify different set of high level parameters (e.g. Multimedia Streaming, VOIP, signaling protocol and file transfer have different parameters and performance indicators). The set of high level QoS requirements of the application will be specified through an Application Level Contract (ALC). These ALCs cannot be passed directly to the admission control and to the scheduler because the underlying resources managed by the scheduler use IEEE $802.11 \mathrm{e}$ protocol parameters (which usually are different by the application ones). The QoS manager acts as a proxy; in particular it translates the high level QoS requirements of the application into the resource allocations, it computes transmission parameters values and it negotiates them with admission control. The set of low level resource requirements produced by QoS manager will be called Resource Level Contract (RLC).

Moreover QoS manager:

- adapts automatically the resource allocation to dynamic changes in the requirements of the application (e.g. when an application wants changes the contract profile, the QoS manager contacts again the admission control and negotiates a new RLC);

- adapts dynamically the resource allocation in order to optimize the resource utilization without sacrificing on QoS requirements;
- maintains as much as possible the resource allocation for each application as close the minimum that is needed to fulfill the ALC.

Finally, in the case an overload occurs (e.g. due to varying network conditions or if a more important QoS request is received), it can decide to change the ALC to degrade the QoS level of one or more applications by a call-back notification so that the application itself can adapt its QoS requirements.

When QoS manager requires admission to the Admission Control the latter computes the theoretical new bandwidth utilization and checks if it is admissible without degradation of preexistent transmissions. The response is sent back to the QoS manager. If the instance request is successful a RLC is established and the QoS manager can communicate transmission parameters to the scheduler.

Before admitting the new flow the Admission Control uses the following admission test:

$$
\sum_{i=1}^{N} \frac{Q_{i}}{P_{i}} \leq U_{l u b}
$$

where $Q_{i} \triangleq C_{i} / r_{i}$ is the average time budget of the medium which is reserved to the $\mathrm{QSTA}_{i}$ within each CAP, $r_{i}$ is the physical bit rate assumed for admission control computations of the $\mathrm{TS}_{i}, C_{i}$ are the bytes transmitted during the $P_{i}$ which is the SI of $\mathrm{TS}_{i}$ and $U_{l} u b$ is least upper bound utilization factor computed for the worst-case available bandwidth.

In all cases if the sum of the bandwidth utilization of the existing reservations, plus the utilization of the new reservation does not exceed $U_{l u b}$, the request is forwarded to the scheduler. If there is not enough bandwidth to serve the new request three different admission control policies exist which act as follows:

- saturation policy, the highest possible budget is assigned to the task so that the total resource utilization does not exceed $U_{l u b}$

- compression policy, in respect of the established ALCs, all the reservation (RLCs) are recomputed ("compressed") so that we can make new space for the new request;

- reject policy, the transmission is rejected.

The Scheduler manages each TS transmission for each admitted QSTA and it assigns dynamically both TXOP and SI to follow the channel variability and streams characteristics. Our scheduler can handle TS with Hard and Soft Real Time guarantees with special regard to VBR flows. VBR flows are supported by assigning TXOP in agreement to the effective temporal demands of QSTA and the length of its queues. The assignment of SI is dynamic, so to poll with greater frequency the stations having in queue traffic with tightening requirements of QoS. It is also able to reclaim the unused time of QSTA which have exhausted their transmission before the end of their TXOP and assign it to the stations which have still useful data to transmit. Delay or advance of the transmission with respect to the pre-agreed rate (in terms of bytes which have been anticipatively used or have not been transmitted by mobile station) are formalized as the scheduling error $\varepsilon_{i}^{(k)}$, defined, at the $k^{\text {th }}$ time 
instant, as the difference between the cumulated bytes to transmit $z_{i}^{(k)} \triangleq k C_{i}^{(k)}$ and the bytes actually transmitted $\bar{z}_{i}^{(k)}$ :

$$
\varepsilon_{i}^{(k)} \triangleq z_{i}^{(k)}-\bar{z}_{i}^{(k)}
$$

The dynamic equation for the evolution of the scheduling error for the $i^{t} h$ real-time data flow is:

$$
\varepsilon_{i}^{(k+1)}=\varepsilon_{i}^{(k)}+C_{i}^{(k)}-\gamma_{i}^{(k)} Q_{i}^{(k)}
$$

where $\gamma_{i}^{(k)}$ is the actual channel speed.

The Predictor estimates the future available bandwidth and the QSTA's queue length, sensing the channel medium and listening to the messages sent by QSTAs. It uses the recent history of these values to correct its estimation. The predictor can be both deterministic and stochastic depending on TS. This information is used by the Feedback System.

The Feedback System senses the effective information acknowledged by QAP and each QSTAs. It also uses the information provided by the predictor to vary transmission parameters of the scheduler in order to respect hard and soft deadlines. It's responsible to minimize the scheduling error. The rapidity of this action can be improved turning on special weights $w_{i}$ for each $\mathrm{TS}_{i}$. The feedback system can compensate little variations of network conditions without the intervention of admission control to establish new RLCs.

During normal condition, if $\sum_{i=1}^{N} \frac{Q_{i}^{(k)}}{P_{i}} \leq U_{l u b}$, feedback system controls the scheduling error assigning:

$$
\forall i, Q_{i}^{(k)} \triangleq \widetilde{Q}_{i}^{(k)}=\frac{C_{i}^{(k)}+\alpha_{i} \varepsilon_{i}^{(k)}}{\rho_{i}^{(k)}}
$$

where $\widetilde{Q}_{i}^{(k)}$ is the required assigned budget to compensate the scheduling error, $\left.\left.\alpha_{i} \in\right] 0,1\right]$ is a fraction of the current scheduling error for each $\mathrm{TS}_{i}$ and $\rho_{i}^{(k)}$ is the predicted channel speed at the physical layer.

During overload condition, if $\sum_{i=1}^{N} \frac{Q_{i}^{(k)}}{P_{i}}>U_{\text {lub }}$, depending the feedback scheme adopted, the allocated budget to each station $i$ is decreased. For example, if the feedback scheme uses a weighted distribution for each $\mathrm{TS}_{i}, \widetilde{Q}_{i}^{(k)}$ is decreased of an amount proportional to the weight $w_{i}$ assigning:

$$
\forall i, Q_{i}^{(k)} \triangleq \widetilde{Q}_{i}^{(k)}-\frac{w_{i} \widetilde{Q}_{i}^{(k)}}{\sum_{j=1}^{N} w_{j} \widetilde{Q}_{j}^{(k)}}\left(\sum_{j=1}^{N} \widetilde{Q}_{j}^{(k)}-U_{l u b} P_{i}\right)
$$

where $\frac{w_{i} \widetilde{Q}_{i}^{(k)}}{\sum_{j=1}^{N} w_{j} \widetilde{Q}_{j}^{(k)}}$ is the percentage of decreasing.

This system can use different feedback schemes according the profile of each TS. By this way the Framework can react to network variations using different compensation models on the basis of the application served.

\section{CONCLUSION}

In this paper we have briefly described MAC-layer access mechanisms provided by 802.11 standard and the QoS enhancements introduced by recent 802.11 e standard. Then we have illustrated the 802.11 e reference scheduler and some alternative schedulers which have been proposed to improve its performance under time-varying network conditions and different traffic specifications. Finally we have proposed the architecture of a novel framework offering better QoS support. This framework lets applications establish contracts with QoS manager that administers the available resources from underlying subsystems. The resulting QoS service is an improvement for applications running over WLAN networks. The adopted scheduler supports real-time applications, variable packet size and variable bit rate traffic streams. Even if the centralized system of HCCA results in the deterministic nature of admission control, we show that many improvements to the reference scheduler can be obtained, especially for VBR streams. Moreover efficient network utilization is also aimed. We have discussed features of this framework showing that it is suitable to be used by software requesting application level contracts, and it is able to manage available resources dynamically under different load conditions.

\section{ACKNOWLEDGEMENTS}

This work has been supported in part by FRESCOR EU project (Contract n. 034026) and by ARTIST2 EU Network of Excellence (IST-004527).

\section{REFERENCES}

[1] Wireless LAN medium access control (MAC) and physical layer (PHY) specification, 1997.

[2] 802.11e-2005 IEEE standard for information technology telecommunications and information exchange between systems local and metropolitan area networks specific requirements part 11: Wireless LAN medium access control (MAC) and physical layer (PHY) specifications: Amendment 8: Medium access control (MAC) quality of service enhancements, 2005.

[3] A. Annese, G. Boggia, P. Camarda, L. A. Grieco, and S. Mascolo. Providing delay guarantees in IEEE 802.11e networks. In 59th IEEE Semiannual Vehicular Technology Conference, VTC Spring, 2004.

[4] P. Ansel, Q. Ni, and T. Turletti. An efficient scheduling scheme for IEEE 802.11e. In Proc. Modeling and Optimization in Mobile, Ad Hoc and Wireless Networks, 2004.

[5] J. Cowling and S. Selvakennedy. A detailed investigation of the IEEE 802.11e HCF reference scheduler for VBR traffic. In 13th International Conference On Computer Communications And Networks (ICCCN 2004), Chicago, US, Oct 2004.

[6] T. Cucinotta, L. Abeni, G. Lipari, L. Marzario, and L. Palopoli. QoS management through adaptive reservations. Real-Time Systems Journal, 29(2-3), Mar 2005.

[7] H. Fattah and C. Leung. An overview of scheduling algorithms in wireless multimedia networks. IEEE Wireless, 9(5):76-83, Oct 2002.

[8] A. Grilo, M. Macedo, and M. Nunes. A service discipline for support of IP QoS in IEEE 802.11 networks. In Proc. PWC 2001, Laapenranta, Finland, Aug 2001.

[9] A. Grilo, M. Macedo, and M. Nunes. A scheduling algorithm for QoS support in IEEE 802.11e networks. IEEE Wireless Communications, pages 36 - 43, jun 2003. 
[10] A. Grilo and M. Nunes. Performance evaluation of IEEE 802.11e. In Proc. PIMRC 2002, volume 1, page 511 517, Lisboa, Portugal, Sep 2002.

[11] S. Lu, V. Bharghavan, and R. Srikant. Fair scheduling in wireless packet networks. IEEE/ACM Trans. Net., 7(4):473 - 489, Aug 1999.

[12] S. Mangold, S. Choi, P. May, O. Klein, G. Hiertz, and L. Stibor. IEEE 802.11e wireless LAN for quality of service. In Proc. European Wireless (EW2002), 2002.

[13] S. Tsao. Extending earliest due-date scheduling algorithms for wireless networks with location dependent errors. In Proc. IEEE VTC. 2000, Boston, MA, Sep 2000.

[14] H. Zhu and I. Chlamtac. An analytical model for IEEE 802.11e EDCF differential services. In Proc 12th International Conference Computer

Communication and Networks, Dallas, TX, Oct 2003. 